\title{
Eingreifende Hermeneutik. Zum Wert hermeneutischen Wissens und Könnens in deutschdidaktischer Hinsicht
}

\author{
Diana Nacarlı · Julia Landgraf · Jörg Kilian
}

Eingegangen: 5. Juli 2021 / Angenommen: 20. Juli 2021 / Online publiziert: 2. November 2021

(C) Der/die Autor(en) 2021

Zusammenfassung Der Beitrag zeigt die Aktualität der Hermeneutik durch ihre Potenziale für einen inklusiven Deutschunterricht. Insbesondere durch das produktive Nicht-Verstehen und die Reflexion (zukünftiger) Lehrender über Verstehensprozesse kann das Potenzial der Hermeneutik deutschdidaktisch genutzt werden.

Schlüsselwörter Hermeneutik · Deutschdidaktik · Inklusion · Lehrer*innenbildung

\section{Intervening Hermeneutics. On the Value of Hermeneutic Knowledge and Skills in German Didactics}

\begin{abstract}
The article shows the topicality of hermeneutics through its potentials for inclusive German teaching. Especially through productive non-understanding and the reflection of (future) teachers on understanding processes, the potential of hermeneutics can be used in German didactics.
\end{abstract}

Keywords Hermeneutics · German Didactics · Inclusion · Teacher Education

\footnotetext{
Diana Nacarlı $(\bowtie) \cdot$ Jörg Kilian

Germanistisches Seminar, Lehrstuhl für Deutsche Philologie/Didaktik der deutschen Sprache, Christian-Albrechts-Universität zu Kiel, Kiel, Deutschland

E-Mail: nacarli@germsem.uni-kiel.de

Jörg Kilian

E-Mail: kilian@germsem.uni-kiel.de

Julia Landgraf

Berlin, Deutschland

E-Mail: julia.landgraf@gmx.de
} 


\section{Inklusive Hermeneutik}

Wenn wir über Hermeneutik reden und die gesellschaftlichen Veränderungen wie Digitalität, die zu einem womöglich veränderten Leseverhalten führen, tun wir das häufig mit Blick auf Leser*innen ohne Förderbedarf, die den Normen bzw. Anforderungen, die in den Bildungsstandards formuliert werden, entsprechen und in bildungspolitischen Diskursen stets mitgedacht werden.

Diese Leser*innen sind in der Lage, Texte zu verstehen und zu entschlüsseln. Sie bringen die kognitiven Voraussetzungen mit, um traditionellen Ansprüchen gerecht zu werden. In der Schule werden ihnen Lerngelegenheiten geboten, um ihre Lesekompetenzen sowie, als Teil derselben, ihre hermeneutischen Kompetenzen zu üben und zu erweitern, um damit den gesellschaftlichen Ansprüchen, die später an sie gestellt werden, zu erfüllen. Leser*innen erweitern damit in der Schule nicht nur ihre Lesekompetenz, sie erarbeiten sich kulturelle Ressourcen und Fachwissen durch das Lesen und vor allem Verstehen von Texten. Dies ist notwendig, damit sie sich ihre selbstbestimmte Teilhabe am gesellschaftlichen Leben sichern können.

Obwohl Bildung und insbesondere Schule den Anspruch haben, inklusiv zu sein, zeigt ein Blick in die empirische Bildungsforschung jedoch schnell, dass neben dem Bildungserfolg im Allgemeinen die Lesekompetenz im Besonderen an Faktoren wie Migrationshintergrund und/oder soziale Herkunft gekoppelt sind (vgl. Hußmann et al. 2017, S. 197). Diese Erkenntnisse sind wichtig, weil sie uns nicht einfach nur zeigen, dass es ein Problem gibt, sondern dass der Zugang zu Wissen und kulturellen Ressourcen Logiken folgt, die aufgrund struktureller Machtverhältnisse große Teile unserer Gesellschaft in ihrer gleichberechtigten Teilhabe behindern und damit exkludierend wirken.

Wenn man sich mit der Krise der Hermeneutik und ihren Symptomen beschäftigt, ist es daher nötig, auch alle Menschen, die in Schulen Lesekompetenz erwerben sollen, um kompetente und mündige Leser*innen zu werden, einzubeziehen. Gleichzeitig müssen wir uns als Menschen, die Teil bildungswissenschaftlicher Institutionen sind, mit unseren eigenen Ansprüchen auseinandersetzen, die wir in einem Begriff von »Lesekompetenz« bündeln. Es sind nicht nur Smartphones oder Digitalität im Allgemeinen, die die Lektürepraxis verändern. Es sind auch die Menschen, die durch die Konzepte Inklusion und Vielfalt einbezogen werden sollen, die ihren berechtigten Platz in der Gesellschaft einfordern und allein durch ihre Präsenz in Räumen wie der Schule eine Veränderung bewirken. Wenn Lesekompetenz, und Textverstehen als Teil derselben, »zu den grundlegenden Qualifikationen gehört [...], um [Schüler*innen] für eine >befriedigende Lebensführung < [...] zu rüsten « ([Hervorhebung im Original] Garbe et al. 2010, S. 10), dann muss sich auch das Nachdenken über Verstehen darauf richten, allen Leser*innen relativ $\mathrm{zu}$ individuellen Bedingungen und Möglichkeiten (Migration, soziale Herkunft, Behinderung) Wege zum Verstehen eines Textes zu bahnen, und Textverstehen inklusiv zu konzipieren.

Eine zentrale Frage, die an dieser Stelle gestellt werden muss, ist: Wo setzen wir an?

Bisher wurde über Lesekompetenztests wie PISA oder IGLU und die daraus resultierende öffentliche Diskussion ein defizitärer Blick auf Schüler*innen selbst und ihren Weg hin zum Verstehen gerichtet. Die Aufgabe bestand dann vor allem darin, 
sie auf einen gewissen Kompetenzstandard zu bringen, um Texte verstehen zu können. Seltener wurden die Texte selbst in den Blick genommen und geprüft, welche Möglichkeiten sie bieten, um verschiedene Zugänge zum Verstehen zu ermöglichen. Versuche in diese Richtung unterliegen darüber hinaus häufig einer Stigmatisierung (siehe Leichte Sprache). Eine inklusive Hermeneutik muss aber mindestens diese beiden am Verstehensprozess Beteiligten in den Blick nehmen: den lesenden Menschen und den zu verstehenden Text. Daraus ergibt sich die Frage: Welche Möglichkeiten bestehen, um Schüler*innen je nach individueller Ausgangslage zu fördern und welche verschiedenen Zugänge zum Verstehen eröffnen die Texte selbst, um daraus Angebote für Schüler*innen zu erzeugen?

Verstehen wird über verschiedene Medien zu erzeugen versucht; um Verstehen jedoch inklusiv zu gestalten, können Impulse aus bereits bestehenden Konzepten gesammelt werden. In Leichte-Sprache-Texte werden bspw. Bilder eingebunden, um das Textverstehen zu erleichtern. Insbesondere Leerstellen, deren Füllung Inferenzen erforderlich machen, können in Texten Verstehenshürden darstellen. Der Einbezug piktoraler Elemente bietet einen empirisch bestätigten Zugang zum Textverstehen, da auf diese Weise Inferenzprozesse, die komplexes Welt- und/oder Handlungswissen voraussetzen, unterstützt werden können. Hermeneutisches Wissen und Können erweisen sich als Voraussetzung für solche Eingriffe in Textverstehensprozesse.

\section{Didaktische Dialektik des (Nicht-)Verstehens}

Eine der wesentlichen Aufgaben der fachdidaktischen Wissenschaften in einer literaten Gesellschaft ist es, zu ergründen, unter welchen Bedingungen und in welcher Weise sprachliche Zeichen eingesetzt werden können, »um einer dem andern etwas mitzuteilen über die Dinge « (Karl Bühler 1934/1982, S. 24, im Anschluss an Platon). >Der eine< und >der andere< mögen zwar das sprachliche Zeichen kennen, verbinden aber Unterschiedliches damit. In Bildungskontexten versuchen die Beteiligten dann, ein gemeinsames Verstehen »über die Dinge« herzustellen. Das kann indes nur partiell erfolgen. Der Bildungsreformer Wilhelm von Humboldt führt aus, dass zwischen den Beteiligten stets »jede noch so kleine Verschiedenheit zittert « (1998, S. 190 f.) und alles Verstehen immer zugleich ein Nicht-Verstehen sei. Der Sprachtheoretiker Hermann Paul setzt diesen Gedanken fort, wenn er notiert, dass eben diese Verschiedenheit »nicht nur die Möglichkeit, sondern die Notwendigkeit des Nichtverstehens« (1920, S. 15) bedinge.

Diese »Notwendigkeit des Nichtverstehens « steht der traditionellen Hermeneutik (Schleiermacher, Dilthey, Gadamer) in gewisser Weise entgegen. Zwar wird ein absolutes Verstehen auch in der traditionellen Hermeneutik nicht erwartet, jedes Nichtverstehen wird aber als ein verunglücktes, mithin gescheitertes Verstehen begriffen. Die traditionelle Hermeneutik ist damit stets positiv ausgerichtet; Nichtverstehen wird von dieser positiven Ausrichtung ausgeschlossen: Es ist negativ; es ist ein Missverstehen; es ist ein Defizit. Gewiss, bei Schleiermacher wird das Missverstehen als sich von selbst ergebende Regularität angesetzt, dem sich das suchende Verstehen des mehr oder minder Feststehenden entgegenstellt. Aber das Missverstehen bleibt doch ein Verstehen, nur eben dass es misslich ist. Missverstehen und 
Nichtverstehen sind indes nicht identisch. Hinzu kommt, dass eine Hermeneutik, die Verstehen als diskursive Rekonstruktion von nicht bereits vorab Feststehendem, sondern immer wieder durch die »Wechselwirkung der Individuen« (Paul 1920, S. 7) neu Erzeugtem erfasst, das Nichtverstehen sehr viel konturierter nicht allein als Voraussetzung, sondern als Bedingung des Verstehens, mithin gar als > Wagenheber $<$ (Tomasello 2003) zum Verstehen konzipieren und didaktisch modellieren kann.

Auch die moderne Didaktik hat allerdings noch einige Schwierigkeiten damit, Nichtverstehen in seiner funktionalen Potenz für das Verstehen zu erfassen und darüber hinaus lerntheoretisch in Beziehung zu setzen zu Wissenserwerb, Wissensvermittlung, Wissenserzeugung. Die Grundsätzlichkeit des Nichtverstehens im Gespräch kann indes als didaktischer Schlüssel bzw. Zugriff genutzt werden, und zwar sogar ohne dass ein einziges >richtiges< Verstehen als Zielpunkt vorhanden sein muss. Ein solcher Zielpunkt kann selbstverständlich vorhanden sein; immerhin soll schulische Bildung das gesellschaftlich als relevant erachtete Wissen vermitteln und an gemeinsam geteilte Sprachzeichen binden, »um einer dem anderen etwas mitzuteilen «. Betrachtet man den Weg dieser Vermittlung als Erzeugung, dann kann das Nichtverstehen (z. B. im Sinne von Piagets >kognitivem Konflikt<) ein bedeutsamer Wagenheber zum Verstehen sein. Für zahlreiche Gegenstände der Bildung und des Lernens existiert indes ein solcher kollektiv geteilter einheitlicher Zielpunkt des Verstehens nicht, zum Beispiel beim literarischen Textverstehen - auch wenn zahllose »Interpretationshilfen« das Gegenteil suggerieren.

Sprache erfüllt eine wichtige, wenn nicht gar die wesentliche Funktion im Rahmen der Bildung des Menschen. Diese Funktion ist bereits im Rahmen unterschiedlicher Theorien zur Beschreibung und Erklärung des menschlichen Lernens, Wissenserwerbs, Bildungsprozesses beschrieben und, zumindest in Ansätzen, empirisch erforscht worden. Bislang kaum berücksichtigt blieb dabei indes, dass, zumal in dialogischen Kontexten der Wissenserzeugung und -vermittlung, ein Nicht- oder Andersverstehen eher der Normalfall ist als ein Verstehen.

»Hermeneutik heute« steht vor diesem Hintergrund vor der Aufgabe, eine sprachtheoretisch und didaktisch fundierte »Theorie produktiven Nichtverstehens« auszuarbeiten. Einen Ausgangspunkt dafür könnte die Rekonstruktion der produktiven Funktionalität von Nichtverstehensdispositionen und Nichtverstehensmodi im LehrLern-Gespräch bilden, namentlich bei ästhetischen Gegenständen. Eine wesentliche Voraussetzung dafür ist die Anerkenntnis unterschiedlichen Verstehens von Etwas als Etwas und im Zusammenhang damit die Anerkenntnis des Nichtverstehens als ein didaktisch zu nutzendes, produktives, methodisches Prinzip, das zu einem Verstehen von Etwas als Etwas führen kann (vornehmlich dort, wo ein Erklären erschwert ist). Eine didaktisch motivierte Diagnostik des Nichtverstehens kann der inklusiven Hermeneutik vorarbeiten, indem sie je individuelle Ansatzstellen für die Nutzung des Nichtverstehens als Wagenheber zum Verstehen ermittelt - und sich als eingreifende Hermeneutik erweist. 


\section{Hermeneutik als Facette professioneller Kompetenz von Deutschlehrer*innen}

Aufbauend auf dem Gedanken, dass das Nicht-Verstehen ein Wagenheber für das Verstehen sein kann, wird ein Blick auf Lehramtsstudierende des Faches Deutsch notwendig. Diese bilden die Schnittstelle zwischen der universitären Germanistik und schulischen Textverstehenssituationen. Befragt man Studierende am Ende ihres Bachelors mit Concept Maps und flankierenden Interviews (vgl. Landgraf 2021), ergeben sich einige Gedankenanstöße sowohl zum Stellenwert der Hermeneutik als auch zum Verständnis von Verstehen und Nicht-Verstehen:

Wenn die Hermeneutik selbst in den Concept Maps erwähnt wird, finden sich Propositionen wie »Hermeneutik $\rightarrow$ verbessert $\rightarrow$ Textverstehen $\ll$. Fragt man Studierende ausgehend von den erstellten Concept Maps nach ihrer Definition von Hermeneutik, wird deutlich, dass zum Teil erhebliche Unsicherheiten bestehen (vgl. Interviewaussage: »[...] Hermeneutik und Strukturalismus hat man immer gehört und man konnte es nie so richtig festlegen« [S3, 31:06]). Ebenso zeigt sich in der Untersuchung, dass das Nicht-Verstehen eben nicht als ein Wagenheber des Verstehens wahrgenommen wird - weder für sich selbst noch in didaktischer Hinsicht. Vielmehr wird die durch das Nicht-Verstehen ausgelöste Unsicherheit entweder überspielt oder auf allen Wegen versucht aufzulösen (vgl. Interviewaussage):

»[...] wenn ich jetzt wirklich merke, ich versteh den Text eigentlich so gar nicht, [...] dann mach ich es meistens so, dass ich versuch den erstmal komplett zu lesen, [...] oder wenn ich ihn so gar nicht versteh oder auch danach immer noch das Gefühl habe, ich habe ihn gar nicht verstanden, gerade bei klassischen Büchern hat man ja doch nochmal die Möglichkeit bspw. auf Wikipedia oder so nachzugucken, was manchmal wirklich gut ist.« (S2, 18:48)

Ansonsten offenbaren sich im Kontext des Textverstehens kognitionswissenschaftliche Wissensbestände wie die Nennung des Construction-Integration-Modells neben Begriffen aus der Lesedidaktik, beispielsweise der 5-Schritt-Lesemethode.

Was kann aus diesen kurzen Ausschnitten der Befragung abgeleitet werden?

Für Lehramtsstudierende scheint die Hermeneutik ein undefiniertes, abstraktes Konstrukt, von dem sie in Lehrveranstaltungen etwas von einer langen Forschungstradition gehört haben und was somit gar nicht unbedingt mit dem in Verbindung gebracht wird, was sie jeden Tag bewusst oder unbewusst tun: reflektiert und regelgeleitet einen individuellen Text verstehen (wollen) (vgl. Detering 2013, S. 190) - und eingreifend ein Textverstehen bei Schüler*innen zu fördern. Diese treffende Minimaldefinition - also Hermeneutik als Kunst des reflektierten und regelgeleiteten Textverstehens - ist aber genau das, was Lehramtsstudierende für sich selbst und ihr späteres Unterrichten bei der Unterrichtsvorbereitung, aber auch bei der Unterstützung der Textverstehensprozesse ihrer Schüler*innen unbedingt benötigen. Dieser Kern hermeneutischen Handelns ist also eine unerlässliche Facette professioneller Handlungskompetenz von Deutschlehrer*innen. Und weiterhin sollten sie augenscheinlich nicht nur von hermeneutischem Handeln wissen, sondern es selbst gelebt und erfahren haben - in ihren privaten Lektüren ebenso wie in denen in universitären Lehrveranstaltungen. Denn auch die immer heterogeneren Studieren- 
den erwerben durch die reflektierte und regelgeleitete Lektüre individueller Texte Hintergrundwissen und Bezugsmöglichkeiten für ihre weiteren Lektüren, die damit nicht nur wiederum leichter verstanden werden können, sondern bei denen die Lust am Lesen und Text sich um ein Vielfaches erweitern kann.

So einfach geschrieben, so schwer umzusetzen. Denn genau diese Angst vor der durch das Nicht-Verstehen ausgelösten Unsicherheit ist wohl eines der größten Hindernisse für ehrliche, gewinnbringende und verstehensorientierte Lektüre. Reine Plädoyers, das Nicht-Verstehen zu schätzen und dessen inhärentes produktives Potenzial zu nutzen, werden wohl ebenso wenig ausreichen wie Plädoyers dafür, anzuerkennen, dass viele Wege und (inklusiv gedacht) viele Fortbewegungsweisen zum (nicht: zu dem einen) Verstehen führen. Es braucht in der universitären Praxis weiterhin und sehr bewusst diese reflektierte und regelgeleitete Lektüre individueller Texte und das Explizieren dieser Praxis durch die Expert*innen der germanistischen Teildisziplinen. Denn nur wenn das immer wieder gelebt wird, werden Studierende im Allgemeinen und Lehramtsstudierende im Besonderen hermeneutisches Denken und Handeln lernen und dies auch in die schulischen Lernsituationen mittragen. Und dabei trägt die Hermeneutik das Potenzial in sich - wenn sie im universitären Studium ausreichend vermittelt und gelebt wird - gerade auch die Heterogenität der Schüler*innen berücksichtigen zu können und somit schon von Beginn an eine durch und durch inklusive Methode zu sein.

Funding Open Access funding enabled and organized by Projekt DEAL.

Open Access Dieser Artikel wird unter der Creative Commons Namensnennung 4.0 International Lizenz veröffentlicht, welche die Nutzung, Vervielfältigung, Bearbeitung, Verbreitung und Wiedergabe in jeglichem Medium und Format erlaubt, sofern Sie den/die ursprünglichen Autor(en) und die Quelle ordnungsgemäß nennen, einen Link zur Creative Commons Lizenz beifügen und angeben, ob Änderungen vorgenommen wurden.

Die in diesem Artikel enthaltenen Bilder und sonstiges Drittmaterial unterliegen ebenfalls der genannten Creative Commons Lizenz, sofern sich aus der Abbildungslegende nichts anderes ergibt. Sofern das betreffende Material nicht unter der genannten Creative Commons Lizenz steht und die betreffende Handlung nicht nach gesetzlichen Vorschriften erlaubt ist, ist für die oben aufgeführten Weiterverwendungen des Materials die Einwilligung des jeweiligen Rechteinhabers einzuholen.

Weitere Details zur Lizenz entnehmen Sie bitte der Lizenzinformation auf http://creativecommons.org/ licenses/by/4.0/deed.de.

\section{Literatur}

\section{Verwendete Literatur}

Bühler, Karl: Sprachtheorie. Die Darstellungsfunktion der Sprache. Mit einem Geleitwort von Friedrich Kainz. Stuttgart/New York: Fischer 1982 (zuerst 1936).

Detering, Heinrich: »Das Gespräch mit den Toten. Bemerkungen über Hermeneutik und Philologie«. In: Gerd Lüer/Horst Kern (Hg.): Tradition, Autonomie, Innovation. Göttinger Debatten zu universitären Standortbestimmungen. Göttingen: Wallstein, 2013, S. 190-213.

Garbe, Christine/Holle, Karl/Jesch, Tatjana: Texte lesen: Lesekompetenz - Lesedidaktik - Lesesozialisation. Stuttgart: Schöningh, 2. Aufl. 2010. 
Humboldt, Wilhelm von: Über die Verschiedenheit des menschlichen Sprachbaues und ihren Einflu $\beta$ auf die geistige Entwicklung des Menschengeschlechts. Hg. von Donatella Di Cesare. Paderborn: Schöningh, 1998.

Hußmann, Anke/Stubbe, Tobias C./Kasper, Daniel: »Soziale Herkunft und Lesekompetenzen von Schülerinnen und Schülern«. In: Anke Hußmann/Heike Wendt/Wilfried Bos/Albert Bremerich-Vos/Daniel Kasper/Eva-Maria Lankes/Nele McElvany/Tobias C. Stubbe/Renate Valtin (Hg.): IGLU 2016. Lesekompetenzen von Grundschulkindern in Deutschland im internationalen Vergleich. Münster/New York: Waxmann, 2017, S. 195-214.

Landgraf, Julia: Verzahnung als Methode, Vernetzung als Ziel. Eine Concept Map-Studie zum Professionswissen im Bereich >Lesen und Textverstehen<. Berlin: Erich Schmidt, 2021.

Paul, Hermann: Prinzipien der Sprachgeschichte. Halle a. S.: Niemeyer, ${ }^{5} 1920$.

Tomasello, Michael: Die kulturelle Entwicklung des menschlichen Denkens. Zur Evolution der Kognition. Aus dem Englischen von Jürgen Schröder. Darmstadt: Wissenschaftliche Buchgesellschaft, 2003, S. 50-54.

\section{Weiterführende Literatur}

Härle, Gerhard/Steinbrenner Marcus: »>Alles Verstehen ist ... immer zugleich ein Nicht-Verstehen.< Grundzüge einer verstehensorientierten Didaktik des literarischen Unterrichtsgesprächs«. In: Literatur im Unterricht 4/2 (2003), S. 139-162.

Hermanns, Fritz/Holly, Werner (Hg.): Linguistische Hermeneutik. Theorie und Praxis des Verstehens und Interpretierens. Berlin/New York: Niemeyer, 2007.

Schnotz, Wolfgang: Aufbau von Wissensstrukturen. Untersuchungen zur Kohärenzbildung bei Wissenserwerb mit Texten. Weinheim: Beltz, 1994.

Wagenschein, Martin: Verstehen lehren. Genetisch - sokratisch-exemplarisch. Mit einer Einleitung von Hartmut von Hentig. Weinheim: Beltz, ${ }^{5} 2010$.

Westerkamp, Dirk: »Inferentielles Nichtverstehen«. In: Internationales Jahrbuch für Hermeneutik 16 (2017), S. 121-138. 